\title{
Dynamic Traffic Prediction with Adaptive Sampling for 5G HetNet IoT Applications
}

\author{
Shuangli Wu $\mathbb{D}^{1,},{ }^{1,2}$ Wei Mao, ${ }^{1,3}$ Cong Liu $\mathbb{D}^{\circ},{ }^{4}$ and Tao Tang ${ }^{4}$ \\ ${ }^{1}$ Computer Network Information Center, Chinese Academy of Sciences, Beijing, China \\ ${ }^{2}$ University of Chinese Academy of Sciences, Beijing, China \\ ${ }^{3}$ Internet Domain Name System Beijing Engineering Research Center, Beijing, China \\ ${ }^{4}$ Beihang University, Beijing, China
}

Correspondence should be addressed to ShuangliWu; wushuangli@cnic.cn

Received 1 April 2019; Accepted 12 June 2019; Published 26 June 2019

Guest Editor: Bo Rong

Copyright (c) 2019 Shuangli Wu et al. This is an open access article distributed under the Creative Commons Attribution License, which permits unrestricted use, distribution, and reproduction in any medium, provided the original work is properly cited.

Due to the proliferation of global monitoring sensors, the Internet of Things (IoT) is widely used to build smart cities and smart homes. 5G HetNets play an important role in the IoT video stream. This paper proposes an improved Call Session Control Function (CSCF) scheme. The improved CSCF server contains additional modules to facilitate IoT traffic prediction and resource reservation. We highlight traffic prediction in this work and develop a compressed sensing based linear predictor to catch the traffic patterns. Experimental results justify that our proposed scheme can forecast the traffic load with high accuracy but low sampling overhead.

\section{Introduction}

The Internet of Things (IoT) makes it possible to connect various physical devices. Vehicles, buildings, etc. embedded in sensors are connected through the Internet of Things, and data can be collected and exchanged via the Internet. The Internet of Things enables mutual communication between devices. In the next decade, the service targets in the Internet of Things will be improved to users in various industries, and the number of machine to machine (M2M) terminals will increase dramatically, and applications will be ubiquitous.

In order to solve the problem of explosive data volume of the IoT, a heterogeneous networks (HetNets) technology is proposed. The problem of $5 \mathrm{G}$ communication is solved by deploying a large number of small cells [1-3]. There are several technical challenges hindering the IoT video streaming over $5 \mathrm{G}$ HetNets. For example, the interactions between HetNet and 5G optical core tremendously depend on the IoT traffic from the local HetNet, which may change periodically for many reasons. Consequently, the system must have a forecast and reservation scheme to dynamically book necessary resource from optical core. In addition, a connection admission control (CAC) mechanism plays an essential role in case the actually required resource overpasses the reserved. Traffic on future fifth-generation (5G) mobile networks is predicted to be dominated by challenging video applications such as mobile broadcasting, remote surgery, and augmented reality, demanding real-time and ultra-high quality delivery. Two of the main expectations of $5 \mathrm{G}$ networks are that they will be able to handle ultra-high-definition (UHD) video streaming and that they will deliver services that meet the requirements of the end user's perceived quality by adopting quality of experience (QoE) aware network management approaches.

To overcome the above challenges, this paper proposes a scheme of improved CSCF server for $5 \mathrm{G}$ HetNets. The improved CSCF can reside itself in a mobile edge computing (MEC) server, with three extra components added to the original, including IoT traffic prediction, bandwidth negotiation, and connection admission control [4].

The improved CSCF first forecasts the IoT traffic load from a local HetNet to the $5 \mathrm{G}$ optical core. After that, it employs Common Open Policy Service (COPS) protocol [5] to adaptively negotiate bandwidth resource. Finally, the connection admission control serves to keep the system in shape and guarantee communication quality.

The IoT is responsible for the communication between different devices, providing better solutions and enhanced 
services for different information flows. Video signal is very important among multimedia services. At the same time, the video traffic on the Internet has increased dramatically, and a lot of research work has been done to improve the viewing experience of the audience.

Video traffic between HetNet and optical core often fluctuates because of use case's characteristic and variation of observed object. Therefore, our proposed improved CSCF server chooses a dynamic mechanism, in a bid to book bandwidth economically. Apparently the traffic prediction component of improved CSCF server has critical impact on the performance of dynamic bandwidth negotiation. In practice, sampling overhead is a real challenge, as traffic predictor must talk to two other components of the improved CSCF server during the sampling process. For instance, traffic predictor needs the calling records from connection admission control component as the input. After the prediction, it has to transfer the result to bandwidth negotiation component, which will further use COPS messages to negotiate with $5 \mathrm{G}$ core network. The procedure above can strongly justify the significance of sampling rate reduction.

Compressed sensing is a promising paradigm that uses signal sparsity to reduce the amount of data that needs to be measured [6, 7]. Compressed sensing (CS) theory indicates that the characteristics of sparse signals are not affected by the signal sparse basis. It can be obtained by a small amount of projection on another basis. The reconstruction of the wideband signal can be obtained by solving the L1 norm optimization. It is concluded that, in the calculation process, the sparsity of the signals in the orthogonal basis determines the minimum projection number and sampling rate required [8].

In the literature, the study of traffic prediction mainly concentrated on the least mean square (LMS) methods that take sampling and prediction as two independent aspects [912]. As a result, the sampling often implements a constant interval and is too prone to low efficiency in IoT environment.

Other than previous work, this paper focuses on the aggregated multiple streams in a HetNet, instead of one separated video stream.

Inspired by compressed sensing theory, we develop an adaptive sampling rate (ASR) linear predictor to overcome the shortcoming of traditional schemes. ASR linear predictor utilizes the prediction error to control sampling interval, so that the sampling and predicting overheads are significantly reduced.

The rest of the paper is organized as the remainder. Section 2 introduces 5G IoT applications in smart cities. Section 3 introduces 5G HetNets and IMS based IoT video streaming. Section 4 develops traffic prediction schemes using adaptive sampling rate and compressed sensing theory. Finally, Section 5 summarizes the advantages of our proposed improved CSCF server and ASR linear predictor.

\section{5G IoT Applications in Smart Cities}

2.1. Smart Cities. The development of the IoTs and cloud computing technology has become an important symbol of our modern information age, especially in the city, providing technical support for the commercial development of the city. The comprehensive and multilevel processing of information technology in the IoTs provides a service platform for data systems. Therefore, in the smart city information system, the digital information processing of the city can timely understand the relevant information of the city.

The use of IoT technology to improve and develop smart city information technology, shield bad information, and guide and support the exchange of useful information enables it to cover all corners of the city in a network and realizes the management and control of the city as a whole, as shown in Figure 1. At the same time, with the help of the intelligent engine and the unified information service system, the management efficiency of urbanization construction has been effectively improved.

For smart cities, their construction and development are inseparable from the IoT communication technology. It can be said that IoT communication technology is an important component of its foundation. One of the important applications is intelligent building management, which is mainly used in the following aspects.

(a) The installation of intelligent building management technology during the construction of the building will enable residents to conveniently manage and control remote items under the cooperation of the IoT.

(b) The establishment of a large public monitoring system: there are many public buildings in the city and there are many equipment and facilities. Therefore, an emergency monitoring system must be established to solve this problem quickly with the assistance of the IoT when unexpected events occur.

(c) Adopt intelligent water, coal, and electricity meters. The RFID chip is built in the table of various facilities, and the data can be collected into the data processor through the table and then transmitted to the background system through the $5 \mathrm{G}$ network to realize the function of automatic meter reading.

(d) Processing of abnormal equipment: remote monitoring of air conditioners, lights, etc. is carried out using wireless sensors and local area network technology during the processing of this problem.

2.2. IoT Intelligent Parking System in Smart City. With the development of economy, there are more and more vehicles in the city, and the problem of parking difficulty is followed. Smart cities can bring effective solutions to this problem. In the smart city, an intelligent parking system based on Internet of Things is proposed. The framework of the system is shown in Figure 2.

This system combines advanced IoT technology, mobile internet technology, and big data technology. As shown in the figure, the system is mainly composed of the video pile, the base station, the server, the mobile client, and the management cloud platform.

The video pile is mainly composed of a camera, a processor, and a communication device. While the system is running, the camera is used to capture images of each parking space and monitor the status of each parking space 


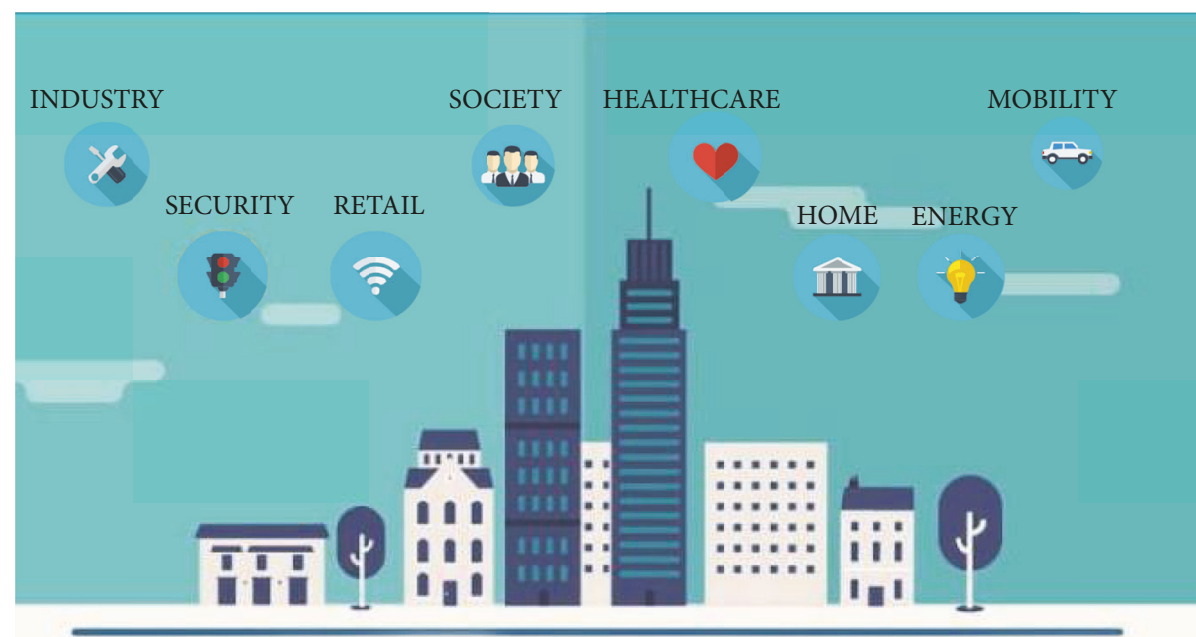

SMART CITY

FIgURE 1: 5G IoT application in smart cities.

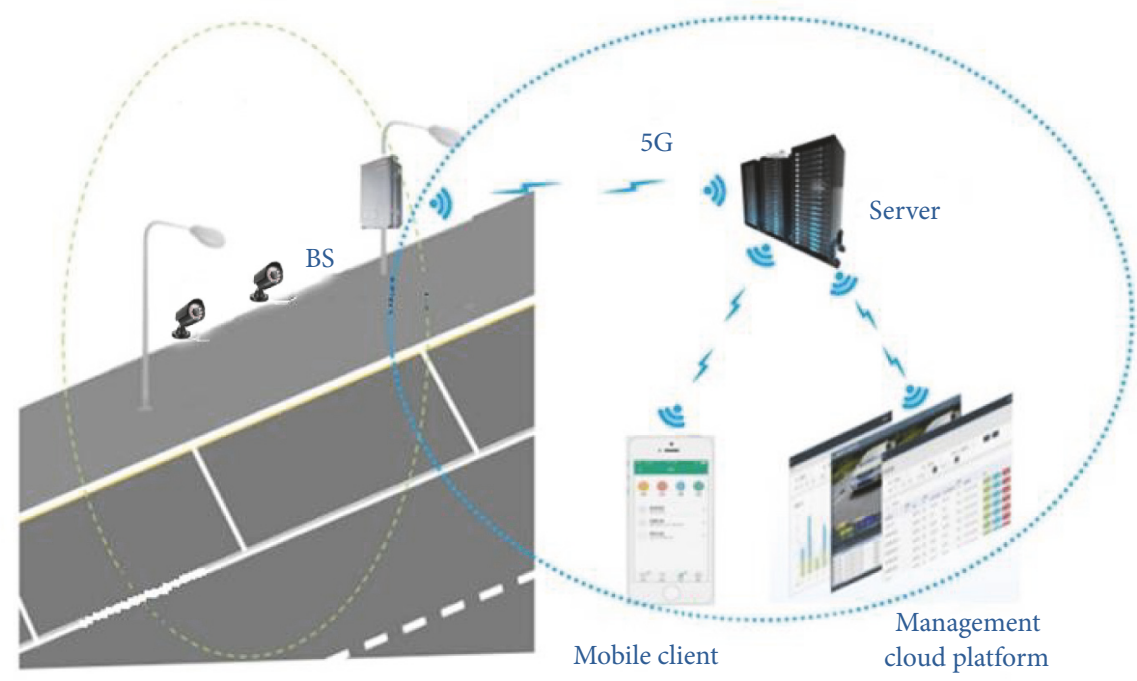

FIGURE 2: Smart parking system.

in real time through target recognition technology. Then, the video pile transmits the information to the base station via the wireless network. The base station forwards the information from different video pile to the server and the cloud management platform. The whole system adopts intelligent management, and the big data technology is adopted in the cloud management platform to carry out regional level parking space scheduling to prevent the problem of uneven resource allocation. The user can query the remaining number of parking spaces and make a parking space reservation in real time through the mobile phone client, and if the user is not familiar with the terrain, the system can recommend the best parking space for the user and provide precise navigation services. Throughout the service process, the system performs automatic billing and supports mobile client online payment.

\section{IoT Video Communication over Multilocation HetNets}

3.1. 5G Core Networks Bridged by MPLS VPN. In the IoTs, HetNets networks consist of eNode BS and low-power nodes (LPNs), a.k.a. access points, a unit that makes it up. Different layers are covered by the two nodes according to the transmission power $[13,14]$. This approach densifies the topology and improves space utilization and spectrum reuse. The structure of the heterogeneous network satisfies the requirements of $5 \mathrm{G}$ technology and greatly improves the spectral efficiency [15]. 


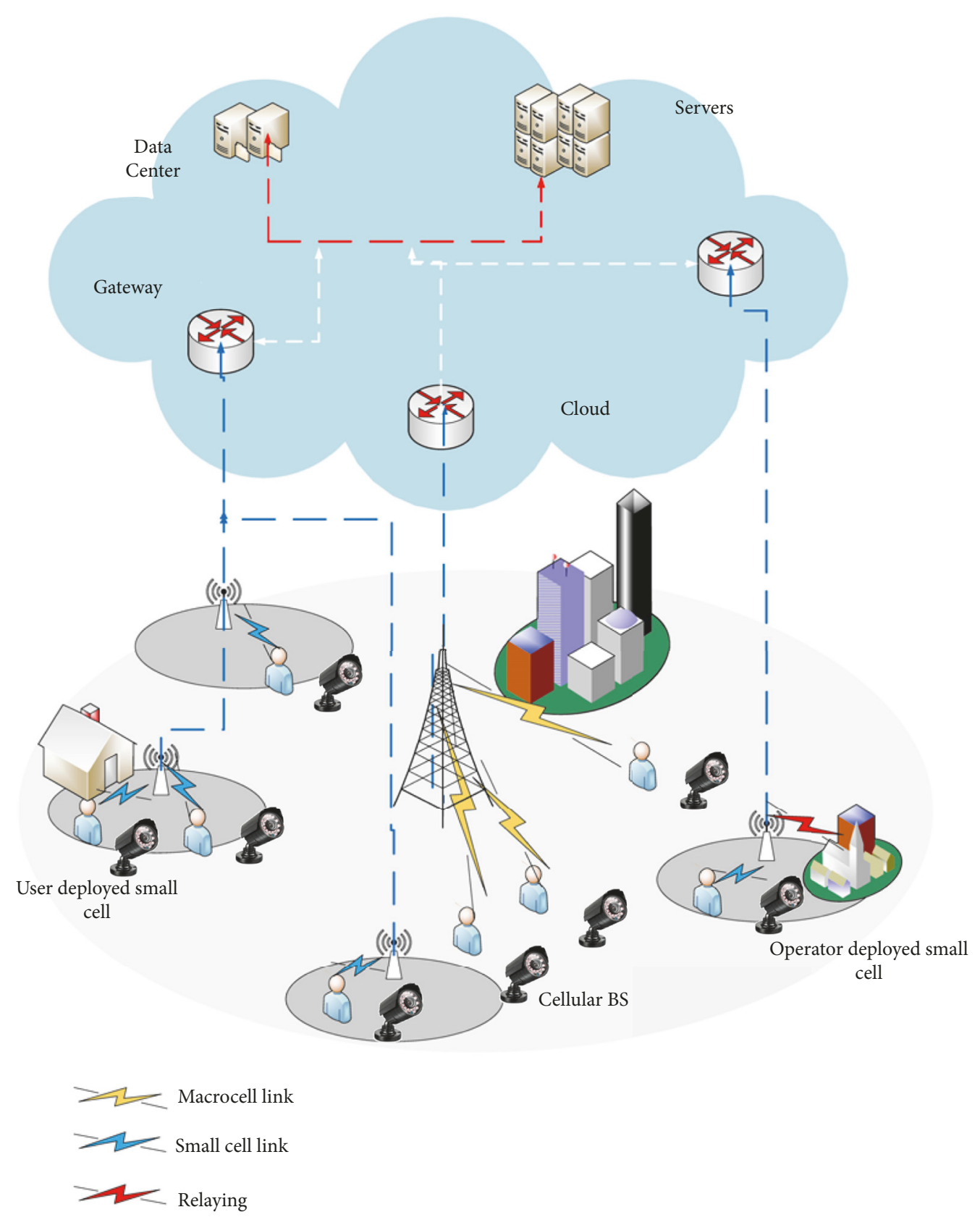

FIgURE 3: A typical 5G IoT heterogeneous network.

In order to solve this problem, 5G wireless networks have received attention and research in order to provide connectivity and meet the requirements of different quality of service (QoS). The key technologies of $5 \mathrm{G}$ enable the deployment of heterogeneous networks (HetNets) to support a large number of IoT data requirements by deploying a large number of small cells.

Network density is the most effective means in many ways to increase network capacity, such as spectrum expansion and spectrum efficiency enhancement [16]. By densely deploying small cells indoors and outdoors, network capacity is increased and network latency is reduced. Small cells include microcells, picocells, and femtocells, which are closer to the user. Figure 3 is a schematic diagram of deployment of a small cell, which can be widely deployed indoors and outdoors, such as in offices, intersections, and plazas. Among them, the data traffic of indoor users can be provided by Wi-Fi. The next generation of Wi-Fi 802.11ac is expected to grow rapidly, providing multigigabit transmission rates. This led to the concept of HetNets, a multilayer network with multiple radio access technologies.

A 5G core network could be enhanced by network slicing, which allows different clients to bridge their private sites over a common provider-owned cloud. Using MPLS or another 


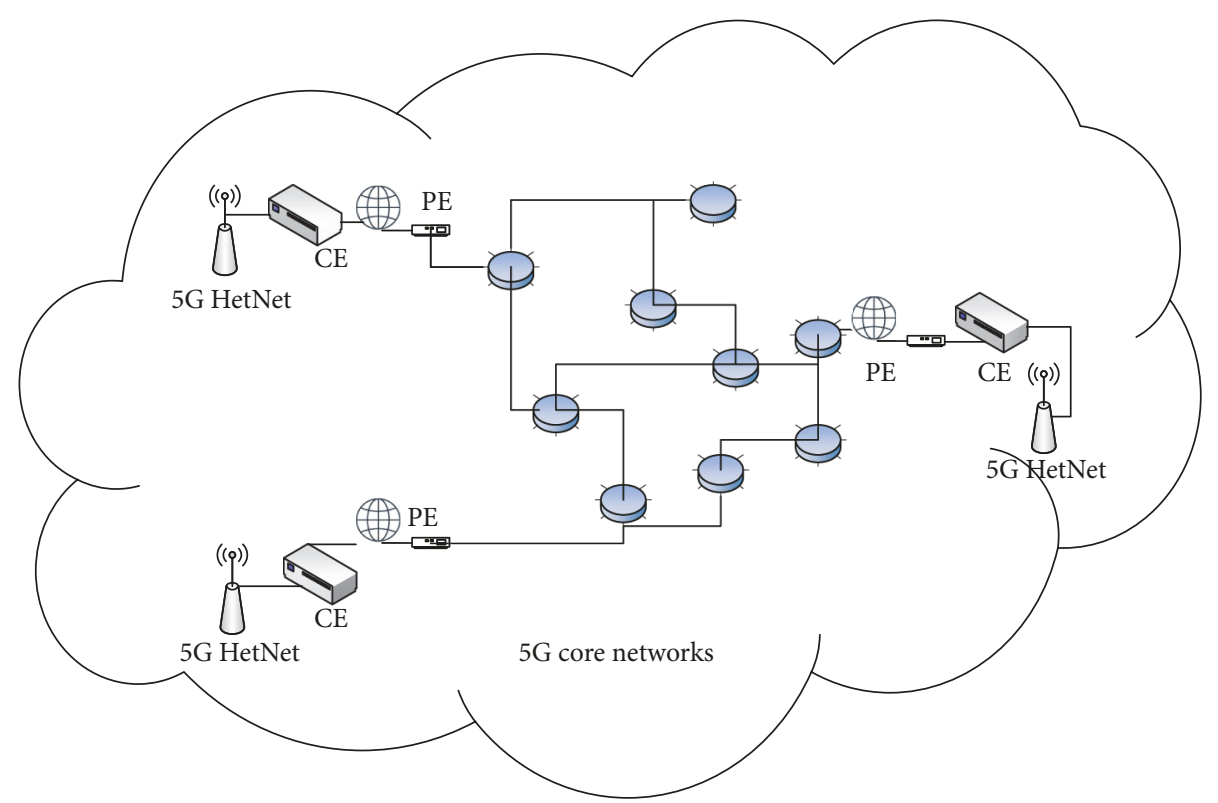

FIGURE 4: Network slicing based 5G core network connecting several local HetNets.

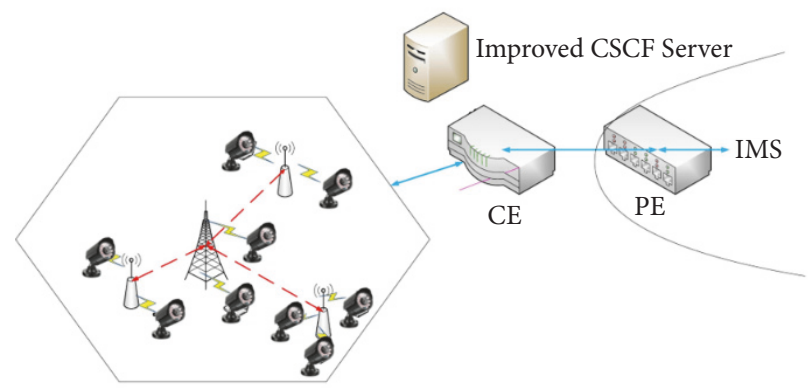

FIGURE 5: IMS based IoT video streaming over 5G HetNets.

similar technology [17], network slicing becomes a novel trend and has a wonderful capability to offer QoS guaranteed services with flexibility. Figure 4 shows an example of network slicing based $5 \mathrm{G}$ core that connects several local HetNets. Using MPLS technology, the core network contains the following types of equipment:

(a) customer edge (CE) router, which serves to connect with client local network directly

(b) provider edge (PE) router, which serves as the interface between $5 \mathrm{G}$ HetNet and optical core

(c) provider $(\mathrm{P})$ router, which serves to forward the user traffic arranged by $\mathrm{CE}$ and $\mathrm{PE}$ routers

3.2. IMS Based IoT Video Streaming. Internet of things (IoT) is regarded recently as the most promising form of the wireless communication environments.

In Internet devices, a large amount of data is generated and accumulated into a large amount of data streams over time. Therefore, higher requirements are imposed on the processing and decomposition of online data. In many applications, a large amount of data storage is generated, such as monitoring the generated video stream data.
5G HetNets transport the video streaming traffic by changing an IoT sensor into an IP Multimedia Subsystem (IMS) terminal. IMS realizes robust IMS service functionality by working closely with Call Session Control Function (CSCF). The CSCF node facilitates Session Initiation Protocol (SIP) to operate session setup and teardown [18].

As a popular video streaming protocol, CSCF conducts signaling and session management and enables connection information to go across network boundaries.

Figure 5 gives an example of IMS based IoT video streaming over $5 \mathrm{G}$ HetNets. The improved CSCF server acts as an intermediate entity that receives and forwards signaling messages. Improved server offers a bunch of crucial functions, such as authentication, authorization, and routing.

3.3. Model of Improved CSCF Server. By definition, the main module of the IMS system is call session control to allow video/voice communication over the convergence of different access networks. It comprises of all the functional modules required to handle all the signaling from end-user to services and other networks. However, conventional CSCF cannot 


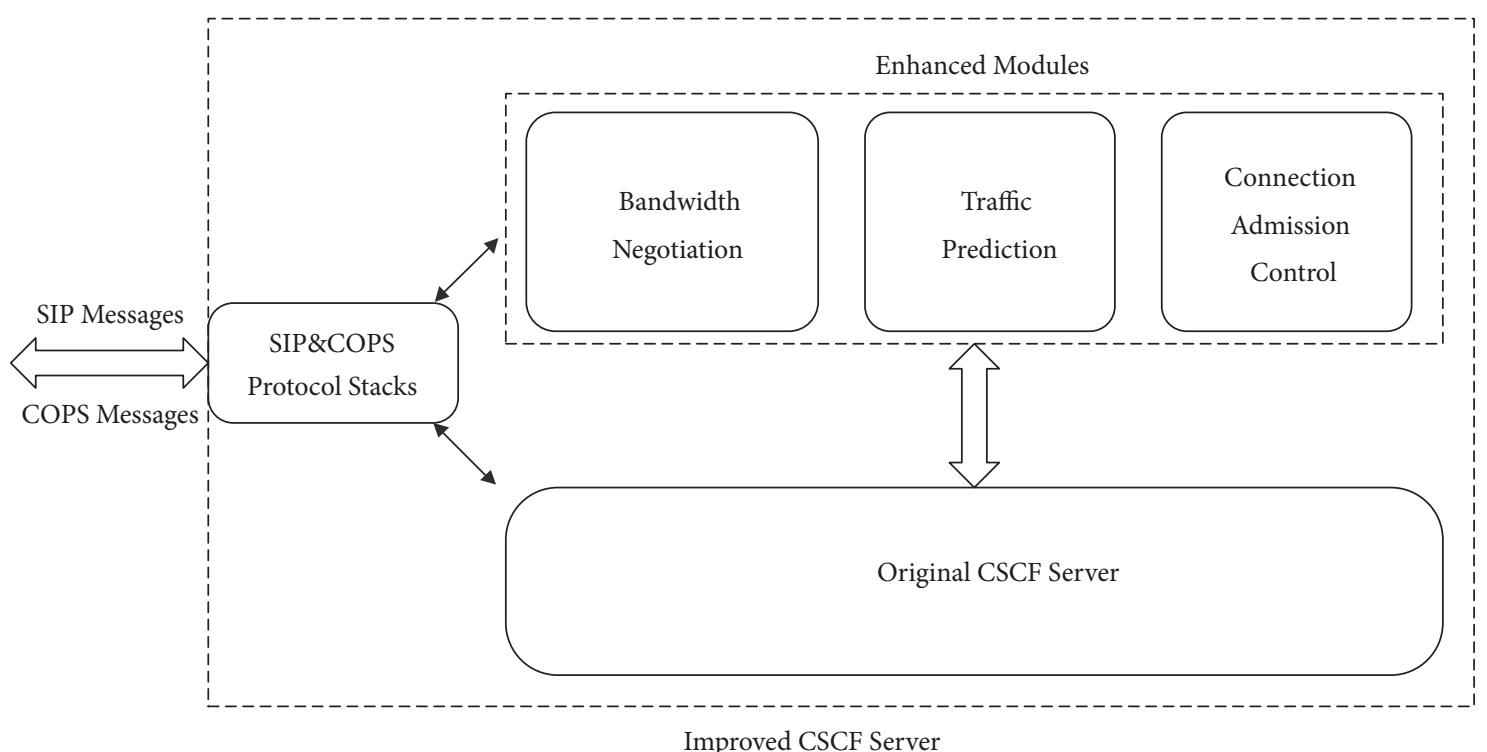

Figure 6: Model of improved CSCF server.

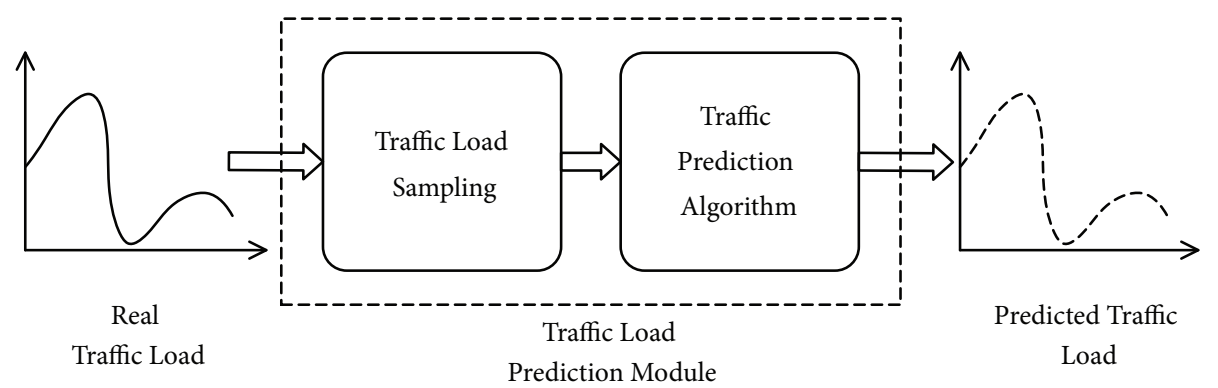

Figure 7: General framework of IoT traffic prediction.

$100 \%$ meet the requirement of IoT video streaming and be modernized.

Figure 6 shows that an improved CSCF server contains three more modules than the original. Our design has the merit that the improved CSCF server bargains with the optical core on behalf of all IoT devices of the local HetNet in advance.

For this reason, the paths required by video streaming are preconstructed before the stream even starts, to reduce the unnecessary signaling delay.

\section{Traffic Prediction in IoT Using Compressed Sensing}

Traffic prediction in the IoT has been widely studied and applied in recent years. Sampling and prediction represent usually two independent parts in the past. For example, most conventional approaches keep the sampling rate unchanged, which may result in low efficiency in IoT video streaming. To overcome this problem, this paper develops a novel compressed sensing based traffic prediction, where error of forecast algorithm goes back to control the sampling interval. Our proposed scheme can significantly reduce the sampling overhead without sacrificing the accuracy.
4.1. Problem Formulation and Architecture Design. Let $B_{n-1: n}$ be the sum of IoT video streaming loads from a local HetNet at time slot $\left[t_{n-1}: t_{n}\right](n=0,1,2, \ldots)$. Let improved CSCF server be exactly aware of the value of $B_{n-1 ; n}$ at time $t_{n-1}$. With this piece of information, it will bargain with $5 \mathrm{G}$ core for the amount of $B_{n-1: n}$ bandwidth using COPS messages. At the next time slot $\left[t_{n}: t_{n+1}\right]$, IoT traffic may change to a new value of $B_{n ; n+1}$, and the improved CSCF server will have to rebargain with $5 \mathrm{G}$ core network again.

It is, nevertheless, impossible for the improved CSCF server to achieve the value of $B_{n-1 ; n}$ before the time of $t_{n}$. Thus, at time $t_{n-1}$, we can estimate the approximate value $B_{n-1: n}$, which is denoted by $B_{n-1: n}^{\prime}$. If $B_{n-1: n}^{\prime}<B_{n-1: n}$, the local HetNet will suffer from the shortage of bandwidth resource to accommodate all video streaming sessions. As a solution, CAC component must reject some of connection requests in order to guarantee overall quality of service.

On the other hand, if $B_{n-1: n}^{\prime}>B_{n-1: n}$, part of bandwidth resource is simply overbooked and useless. In this sense, the traffic prediction is critical to ensure a good performance of our proposed scheme.

Figure 7 presents the general framework of IoT traffic prediction module with the component of traffic load sampling and the component of traffic prediction algorithm. 
We will give more elaboration on these two components next.

4.2. IoT Traffic Sampling. An IoT device initiates a video stream session by sending an INVITE message to the improved CSCF server, in a bid to indicate called URL and bandwidth requirement. The improved CSCF server saves every request in the record for IoT traffic sampling usage, regardless the request is accepted by $\mathrm{CAC}$ or not.

In this paper, we use $B_{n-1: n}$ to represent the average IoT traffic interval $\Delta t_{n-1 ;} n=t_{n}-t_{n-1}$. In practice, the system achieves $B_{n-1: n}$ from the record in the improved CSCF server. Conventional approach conducts traffic sampling with constant rate, which rules

$$
\Delta t_{0,1}=\Delta t_{1,2}=\cdots=\Delta t_{n-1, n}=\ldots, \quad(n=0,1,2, \ldots)
$$

Let $B(t)$ be the traffic load function varying with time t, and let $B(f)$ be the frequency counterpart of $B(t)$ with the upper bound of $f_{\max }$. Nyquist theorem regulates the idea that sampling rate must be at least twice of $f_{\text {max }}$ to prevent aliasing [19]. In IoT video streaming, $f_{\max }$ could be high in some complicated scenarios, which make constant sampling rate not applicable at all.

4.3. Compressed Sensing. Compressed sensing theory is a theory that has been widely used in recent years and is widely used in signal processing. It is also called compressed sampling or sparse sampling. CS wants to sample the signal at a sampling rate much lower than Shannon's Law and find a solution for underdetermined linear systems while maintaining the basic information of the signal. [20-24].

In order to implement the compressed sensing theory, the following solution can be used to estimate the sparse vector $\mathrm{x} \in \mathbb{C}^{N}$ using the observed measurement vector $y \in \mathbb{C}^{M}$. A method based on measurement equations is applied. The formula is as follows:

$$
y=\Phi x+w
$$

The measurement matrix is represented by $\Phi \in \mathbb{C}^{M \times N}$. $w \in \mathbb{C}^{N}$ represents the unknown vector of measurement noise and modeling error. The reconstruction process can only occur under ideal conditions, i.e., only when the signal $\mathrm{x}$ is $\mathrm{S}$ sparse $(S \ll N)$. That is, there can be at most $\mathrm{S}$ nonzero items. During the operation, the number of observations is significantly smaller than the number of variables, i.e., $\gg M$.

In practice, the signal we are dealing with is not a sparse signal. The processing method in this time is to express $\{\Psi\}_{i=1}^{N}$ on some basis with the corresponding sparse coefficient $\theta_{i}$. Processing equation (1) yields

$$
y=\Phi x+w=\Phi \Psi \theta+w=\Theta \theta+w
$$

where $\theta \in C^{L}$ is a $L$ dimension sparse vector of coefficients based on the basis matrix $\Psi \in C^{N \times L} . \Theta=\Phi \Psi$ is a $M \times L$ matrix $(M \ll L)$.

However, because fewer measurements are applied than the entries in $\theta$, the resulting solution is uncertain. In order to recover the $S$ sparse signal, $l_{0}$ norm of the most sparse sequence should be found from all feasible solutions. Minimization can be used for the reconstruction process. The formula is expressed as follows:

$$
\begin{aligned}
\widehat{\theta}=\min & \|\theta\|_{0} \\
\text { s.t. } & \|\Theta \theta-y\|_{2} \leq \Xi
\end{aligned}
$$

where $\hat{\theta}$ refers to the estimated vector for $\theta$ and $\|\mathrm{w}\| \leq \Xi$ is noise tolerance. However, for practical operations, the $l_{0}$ norm minimization has huge computational complexity, and a reconstruction algorithm with lower computational cost must be developed.

Studies have shown that the reconstruction process of signal $x$ must satisfy a condition that matrix $\Theta$ cannot map two different $S$ sparse signals to the same set of samples. Therefore, the matrix $\Theta$ must satisfy the restricted equidistance property (RIP) $[25,26]$.

4.4. Adaptive Sampling Rate. (a) The definition of traditional compressive sensing: sparse signals can be recovered from a small number of nonadaptive linear measurements. Let signal $x$ be $K$ sparse in basis/dictionary $\Psi$. For example, $\Psi$ is DFT matrix, if the signal is frequency domain sparse. $\Psi$ can be expressed as a matrix as shown in Figure 8(a). In Figure 9, if signal $x$ is sparse, then we can use compressive sensing by $y=\Phi \times x$

$\Phi$ is the measurement matrix and the theory of CS states that random $\Phi$ will work. How to understand this? For example, if the signal is sparse in frequency domain, then we make a few random samplings in time domain. In contrast, if the signal is sparse in time domain, we make a few random samplings in frequency domain.

(b) Address the situation that the appearance time of sparse signal can be predicted. In this case, $\Psi$ is identity matrix I as shown in Figure 8(b). Then, measure matrix $\Phi$ is part of $\mathrm{I}$, which involves the time points where the sparse signal appears. $\Phi$ is not random anymore. In practice, we propose variable sampling rate scheme to predict these time points (or the measurement matrix).

If a fixed sampling interval applies, as mentioned before, the sampling rate must double the highest frequency of traffic curve to achieve accurate prediction.

Accordingly, it is significant to develop an inconstant sampling rate scheme with much lower overhead.

Variable sampling rate comes from the observation of the traffic curve in Figure 10, with combination of slow and fast changing periods. The fluctuation of traffic significantly depends on the timely feature of IoT devices. For example, the cameras monitor the parking cars' moving and stopping according to people's behavior, which is obviously related to the number of car arrivals and departures. In Figure 8, fast changing zone represents the period of rapid event varying, such as morning peak hour; slow changing zone represents the period of steady event numbers, such as at night.

With the goal of reducing the sampling overhead, a straightforward adaptive rate approach is to utilize low rate in slow changing period and high rate in fast changing 


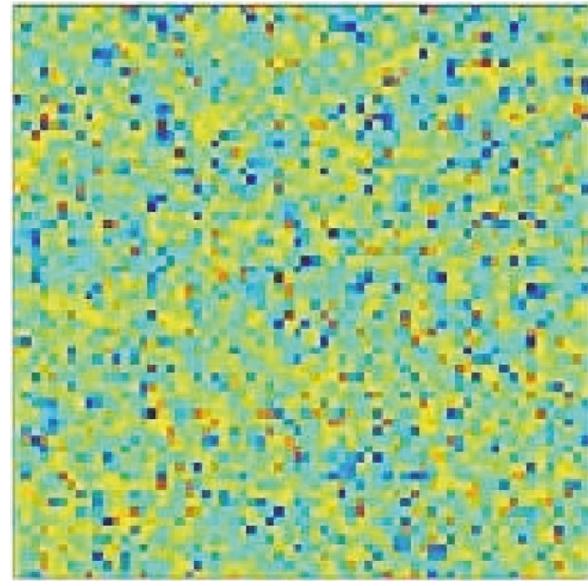

(a) DFT matrix

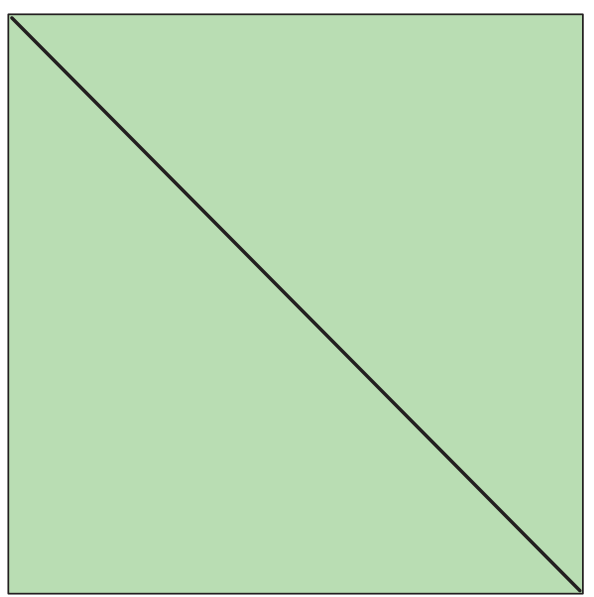

(b) Identity matrix I

Figure 8: The matrix involved in the compressive sensing.

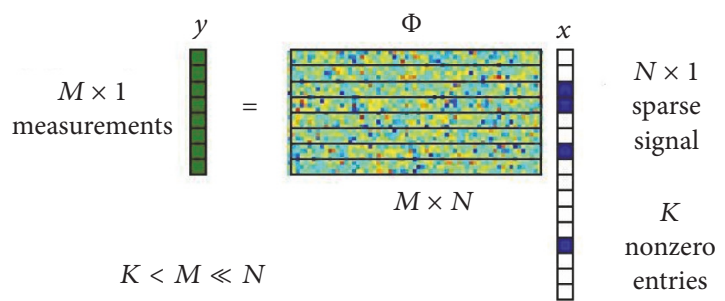

FIGURE 9: The process of compressive sensing.

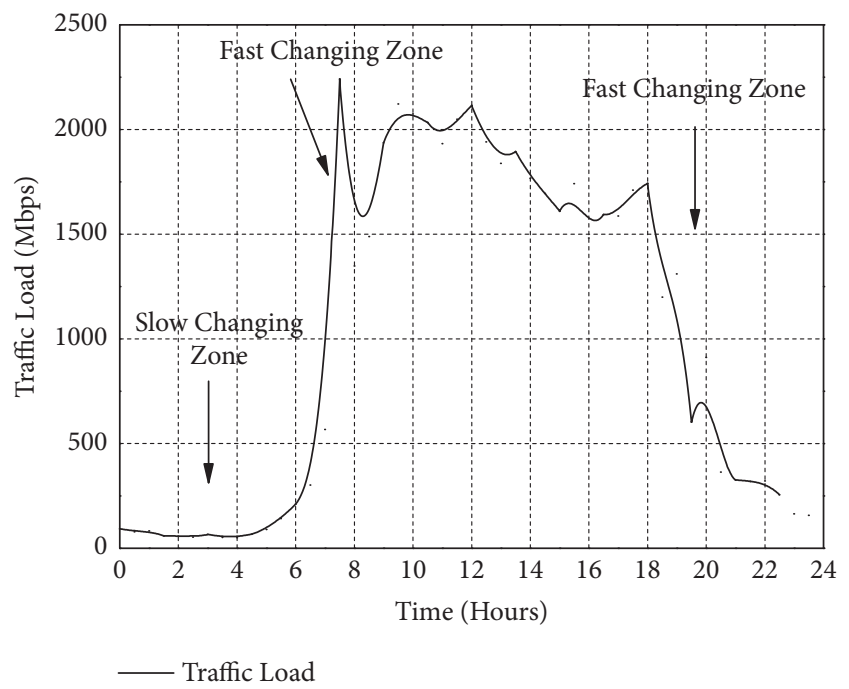

FIGURE 10: IoT traffic load curve of a parking monitor camera.

period. The adaptive sampling interval aims to sample and reconstruct the traffic curve efficiently and effectively, while keeping the same prediction accuracy as constant rate approach does.
Next, we study a typical scenario as follows. Let's divide an IoT traffic curve into a number of slow changing and fast changing periods by using $f_{\max }^{s}$ and $f_{\max }^{f}$ to represent the maximum frequencies, respectively. Nyquist sampling theory states that the sampling rate must be greater than $2 f_{\max }^{s}$ in case of slow changing zone and $2 f_{\max }^{f}$ in case of fast changing. After that, we can achieve the average rate of sampling $R_{V S R}^{\text {avg }}$ by

$$
R_{V S R}^{a v g}=\frac{2 f_{\max }^{s} T_{s}+2 f_{\max }^{f} T_{f}}{T_{s}+T_{f}}
$$

where $T_{s}$ and $T_{f}$ are time of slow and fast changing. Since $f_{\max }^{s}<f_{\max }^{f}<f_{\max }$, we have $R_{V S R}^{\text {avg }}<2 f_{\max }$, implying that ASR approach has much less sampling overhead than its constant counterpart.

4.5. Traditional Linear Predictor. The traditional predictor used most often in practice is the least mean square (LMS) linear filter. A k-step LP makes a guess of the value of $x(n+k)$ by a linear sum of the current and past $\mathrm{x}(\mathrm{n})$. Mathematically, the pth-order k-step is given by

$$
\widehat{x}(n+k)=\sum_{i=0}^{p-1} w_{i} x(n-i)=w^{T} x(n)
$$

where $\widehat{x}(n+k)$ is the estimated value of $x(n+k), w=$ $\left[w_{0}, w_{1}, \ldots, w_{p-1}\right]^{T}$ is the weights, and $x(n)=[x(n), x(n-$ $1), \ldots x(n-p+1)]^{T}$ is priori knowledge. We further define the prediction error as

$$
e(n)=x(n+k)-=\widehat{x}(n+k)-w^{T} x(n)
$$

Then, LP updates $w(n)$ using the following recursive equation:

$$
\mathrm{w}(n+1)=\mathrm{w}(n)+\frac{\mu e(n) \mathrm{x}(n)}{\|\mathrm{x}(n)\|^{2}}
$$

where $\mu$ is the step size that may keep constant (constant step size (CSS)) or adaptive (adaptive step size (ASS)). 


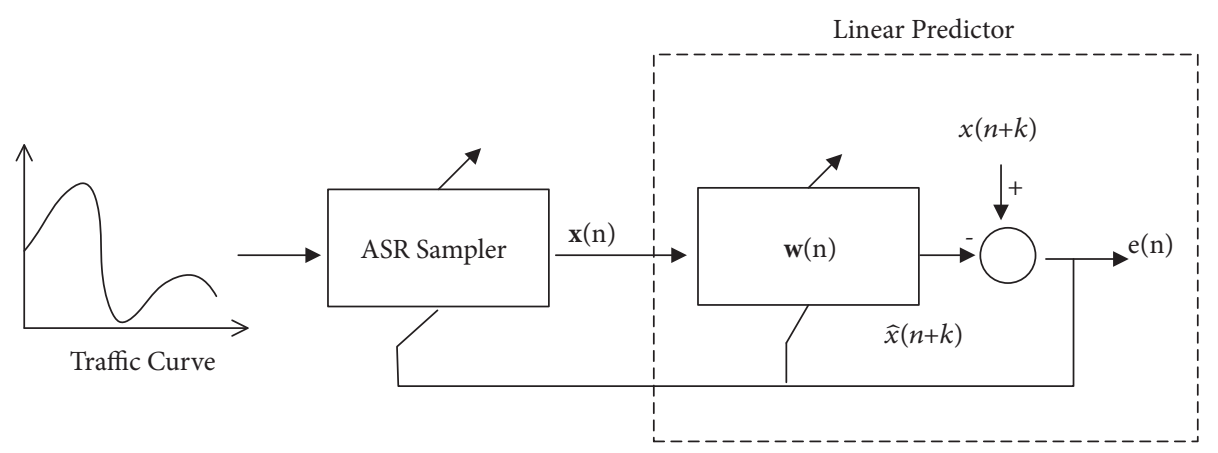

FIGURE 11: ASR-LP working with variable sampling rate.

4.6. Adaptive Sampling Rate Linear Predictor. Realistically the traffic curve is unknown at the time of prediction, and thus it is a mission impossible to chop it into slow and fast changing periods. We, therefore, develop an adaptive prediction scheme ASR-LP, as shown in Figure 11.

ASR-LP rules the sampling rate at time $t_{n}$ as $R s(n)=$ $1 / \Delta t_{n}-1$; and then update it in integration by

$$
R_{s}{ }^{\prime}(n+1)=R_{s}(n)\left[q+(1-q) \frac{|e(n)|}{E_{b}}\right]
$$

with $0<q<1, E_{b}>0$, and

$$
R_{s}(n+1)= \begin{cases}R_{s}^{\max }, & \text { if } R_{s}^{\prime}(n+1)>R_{s}^{\max } \\ R_{s}^{\min }, & \text { if } R_{s}^{\prime}(n+1)<R_{s}^{\min } \\ R_{s}{ }^{\prime}(n+1), & \text { otherwise }\end{cases}
$$

where $0<q<1$ is the remembering factor, Eb is the targeted prediction error bound, and $R s_{\max }$ and $R s_{\max }$ are the upper and lower bounds of sampling rate.

The scheme of ASR-LP optimizes sampling interval by the following principal. The larger the prediction error is, the sharper the traffic curve changes. As a result, we must increase the sampling rate for satisfying prediction accuracy. In contrast, the smaller the prediction error is, the lower the sampling rate goes. Particularly, network operator can determine the value of targeted prediction error bound Eb. If $|e(n)|>E_{b}$, the sampling rate should be increased; if $|e(n)|<$ $E_{b}$, the sampling rate should be decreased; if $|e(n)|=E_{b}$, the sampling rate should be kept unchanged.

In addition to $\mathrm{Eb}$, there are still three other parameters in (9) and (10); i.e., $R s_{\max }$ is the upper bound of sampling rate, which can lead to lower prediction error but larger computational complexity as the value rises. $R s_{\min }$ is the lower bound of sampling rate, which represents the bottom line responding time of a linear predictor. Last but not least, $\mathrm{q}$ decides how much the current sampling rate is relying on its previous value rather than the prediction error. We have to leverage the value of remembering factor $q$ for the optimal performance of predictor, with $q=70 \%$ as a typical case.

In our research, we have conducted simulation studies on a variety of traffic curves to evaluate the performance

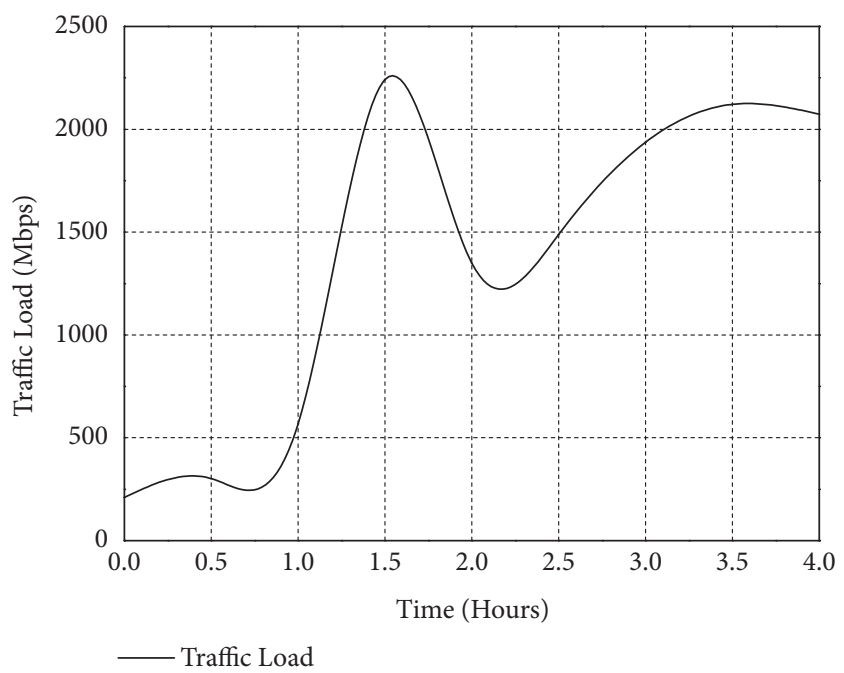

FIgURE 12: IoT Traffic load of four hours for parking monitor camera.

of our proposed ASR-LP scheme. Due to the limit of space, we demonstrate only one typical curve in Figure 10 as an example. However, the simulation results from this example are also largely true to the general cases.

Figure 10 is collected from Melbourne on street car parking sensor data [26], which reflects the typical timing feature of IoT users. It is worth noting that the curve of IoT traffic is dramatically different from previously investigated mobility models in existing literature, such as Brownian motion model.

We use Figure 12 as prototype traffic curve to compare the performance of three schemes as shown in Figure 12. In addition to its advantage in the running time, VSR-NLMS does have another important virtue, i.e., a lower average sampling rate compared to FSS-NLMS and VSS-NLMS. It shows that, with the same simulation configuration as shown in Figure 12, VSR-NLMS achieves an average sampling rate of $1 / 371 \mathrm{~Hz}$, which is lower than $1 / 420 \mathrm{~Hz}$ for FSS-NLMS and VSS-NLMS. Clearly, VSR-NLMS has the lowest sampling rate and thus the least sampling overhead.

Figure 13 reveals that ASR-LP can basically tie ASS-LP, but far outperform CSS-LP regarding prediction accuracy. 


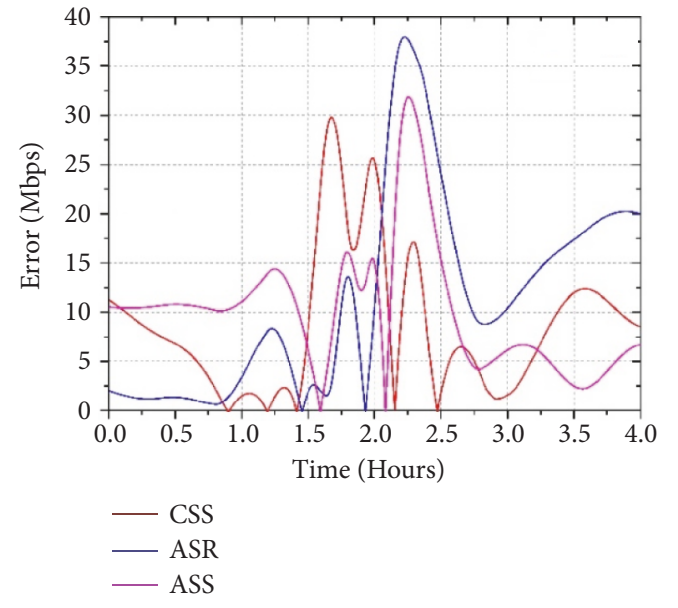

FIGURE 13: Errors of different predicting methods in four hours.

$A S R-L P: E b=100 M b p s, R s_{\max }=1=300 H z, R s_{\min }=$ $1 / 800 \mathrm{~Hz}, \operatorname{Rs}(0)=1 / 420 \mathrm{~Hz}$, and $q=70 \%$.

$C S S-L P: p=4, \mu=0.1, A S S-L P: \mu_{\max }=0.25, \mu_{\min }=$

Low overhead of average sampling is a significant advantage of ASR-LP, compared to its counterpart. Intensive simulation study reveals that ASR-LP can save at least half sampling rate over constant sampling rate linear predictors with the same accuracy.

\section{Conclusions}

This paper studies the deployment of IoT video streams on 5G HetNets and proposes an improved CSCF server solution to promote IoT traffic prediction and resource reservation. We further designed a linear predictor based on compressed sensing to capture traffic patterns, greatly reducing sampling overhead and computational complexity. Intensive analysis and simulation results show that the proposed scheme can effectively improve performance and save time and cost.

\section{Data Availability}

The data used to support the findings of this study are included within the article.

\section{Conflicts of Interest}

The authors declare that they have no conflicts of interest.

\section{References}

[1] M. Li, Y. Du, X. Ma, and S. Huang, "A 3-param network selection algorithm in heterogeneous wireless networks," in Proceedings of the IEEE 9th International Conference on Communication Software and Networks (ICCSN '17), pp. 392-396, IEEE, Guangzhou, China, May 2017.

[2] G. Ma, Y. Yang, X. Qiu, Z. Gao, and H. Li, "Fault-tolerant topology control for heterogeneous wireless sensor networks using Multi-Routing Tree," in Proceedings of the 15th IFIP/IEEE International Symposium on Integrated Network and Service Management (IM '17), pp. 620-623, Lisbon, Portugal, May 2017.

[3] S. Han, Y. Huang, W. Meng, C. Li, N. Xu, and D. Chen, "Optimal power allocation for SCMA downlink systems based on maximum capacity," IEEE Transactions on Communications, vol. 67, no. 2, pp. 1480-1489, 2019.

[4] A. Montazerolghaem, M. H. Moghaddam, and A. Leon-Garcia, "OpenSIP: toward software-defined SIP networking," IEEE Transactions on Network and Service Management, vol. 15, no. 1, pp. 184-199, 2018.

[5] A. K. Vimal, S. Pandit, A. K. Godiyal, S. Anand, S. Luthra, and D. Joshi, "An instrumented flexible insole for wireless COP monitoring," in Proceedings of the 8th International Conference on Computing, Communications and Networking Technologies (ICCCNT '17), pp. 1-5, Delhi, India, July 2017.

[6] A. Mirrashid and A. A. Beheshti, "Compressed remote sensing by using deep learning," in Proceedings of the 9th International Symposium on Telecommunications (IST '18), pp. 549-552, IEEE, Tehran, Iran, December 2018.

[7] S. Rouabah, M. Ouarzeddine, and B. Souissi, "SAR images compressed sensing based on recovery algorithms," in Proceedings of the 2018 IEEE International Geoscience and Remote Sensing Symposium (IGARSS '18), pp. 8897-8900, IEEE, Valencia, Spain, July 2018.

[8] S. Han, Y. Zhang, W. Meng, and H. Chen, "Self-interferencecancelation-based SLNR precoding design for full-duplex relayassisted system," IEEE Transactions on Vehicular Technology, vol. 67, no. 9, pp. 8249-8262, 2018.

[9] S. Han, S. Xu, W. Meng, and C. Li, "Dense-device-enabled cooperative networks for efficient and secure transmission," IEEE Network, vol. 32, no. 2, pp. 100-106, 2018.

[10] D. Ghadiyaram, J. Pan, and A. C. Bovik, "A subjective and objective study of stalling events in mobile streaming videos," IEEE Transactions on Circuits and Systems for Video Technology, vol. 29, no. 1, pp. 183-197, 2019.

[11] Z. Zheng, L. Pan, and K. Pholsena, "Mode decomposition based hybrid model for traffic flow prediction," in Proceedings of the 3 rd IEEE International Conference on Data Science in Cyberspace (DSC '18), pp. 521-526, IEEE, Guangzhou, China, June 2018.

[12] S. Fan and H. Zhao, "Delay-based cross-layer QoS scheme for video streaming in wireless ad hoc networks," China Communications, vol. 15, no. 9, pp. 215-234, 2018.

[13] T. Zhang, L. Feng, P. Yu, S. Guo, W. Li, and X. Qiu, "A handover statistics based approach for cell outage detection in self-organized heterogeneous networks," in Proceedings of the 15th IFIP/IEEE International Symposium on Integrated Network and Service Management (IM '17), pp. 628-631, IEEE, Lisbon, Portugal, May 2017.

[14] S. Sun, M. Kadoch, L. Gong, and B. Rong, "Integrating network function virtualization with SDR and SDN for 4G/5G networks," IEEE Network, vol. 29, no. 3, pp. 54-59, 2015.

[15] M. Lu, Z. Qu, Z. Wang, and Z. Zhang, "Hete_MESE: multidimensional community detection algorithm based on multiplex network extraction and seed expansion for heterogeneous information networks," IEEE Access, vol. 6, pp. 73965-73983, 2018.

[16] Z. Zhang, L. Song, Z. Han, and W. Saad, "Coalitional games with overlapping coalitions for interference management in small cell networks," IEEE Transactions on Wireless Communications, vol. 13, no. 5, pp. 2659-2669, 2014. 
[17] K. Zhang, S. Leng, Y. He, S. Maharjan, and Y. Zhang, "Cooperative content caching in 5G networks with mobile edge computing," IEEE Wireless Communications Magazine, vol. 25, no. 3, pp. 80-87, 2018.

[18] J. Rosenberg, H. Schulzrinne, G. Camarillo et al., "SIP: session initiation protocol," RFC 3261, IETF, June 2002.

[19] K. Zhang, S. Leng, Y. He, S. Maharjan, and Y. Zhang, "Mobile edge computing and networking for green and low-latency internet of things," IEEE Communications Magazine, vol. 56, no. 5, pp. 39-45, 2018.

[20] G. Shrividya and S. H. Bharathi, "A study of optimum sampling pattern for reconstruction of MR images using compressive sensing," in Proceedings of the Second International Conference on Advances in Electronics, Computers and Communications (ICAECC '18), pp. 1-6, Bangalore, India, Feburary 2018.

[21] Y. Wu, B. Rong, K. Salehian, and G. Gagnon, "Cloud transmission: a new spectrum-reuse friendly digital terrestrial broadcasting transmission system," IEEE Transactions on Broadcasting, vol. 58, no. 3, pp. 329-337, 2012.

[22] A. Sammoud, A. Kumar, M. Bayoumi, and T. Elarabi, "Realtime streaming challenges in Internet of Video Things (IoVT)," in Proceedings of the 50th IEEE International Symposium on Circuits and Systems (ISCAS '17), pp. 1-4, Baltimore, Md, USA, May 2017.

[23] N. Eswara, K. Manasa, A. Kommineni et al., "A continuous QoE evaluation framework for video streaming over HTTP," IEEE Transactions on Circuits and Systems for Video Technology, vol. 28, no. 11, pp. 3236-3250, 2018.

[24] B. Rong, Y. Qian, K. Lu, H.-H. Chen, and M. Guizani, "Call admission control optimization in WiMAX networks," IEEE Transactions on Vehicular Technology, vol. 57, no. 4, pp. 25092522, 2008.

[25] I. D. Irawati, A. B. Suksmono, and I. Y. M. Edward, "Local interpolated compressive sampling for internet traffic reconstruction," in Proceedings of the International Conference on Advanced Computing and Applications (ACOMP '17), pp. 93-98, Ho Chi Minh City, Vietnam, December 2017.

[26] N. Anselmi, L. Poli, G. Oliveri, and A. Massa, "Three dimensional imaging with the contrast source compressive sampling," in Proceedings of the International Applied Computational Electromagnetics Society Symposium - China (ACES '18), pp. 1-2, IEEE, Beijing, China, July 2018. 


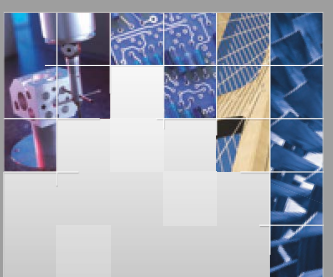

\section{Enfincering}
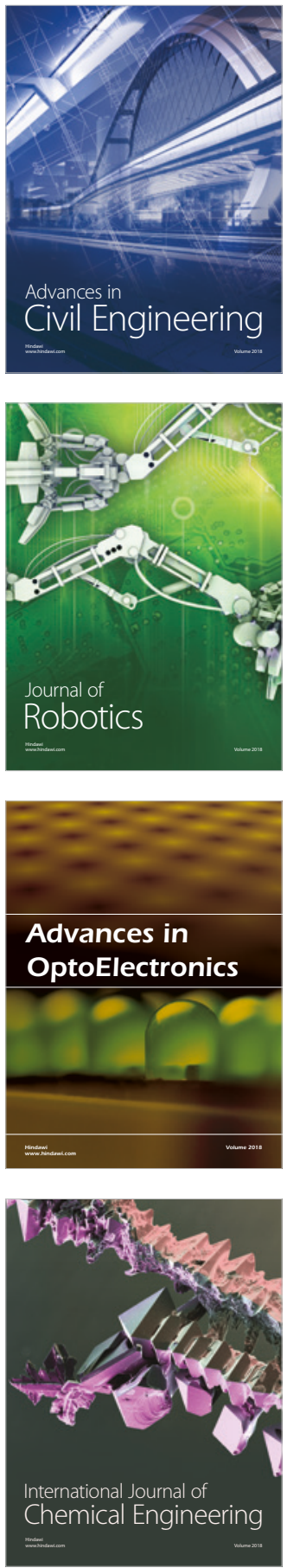

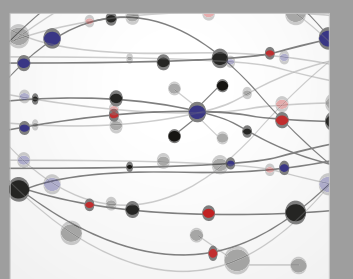

\section{Rotating \\ Machinery}

The Scientific World Journal

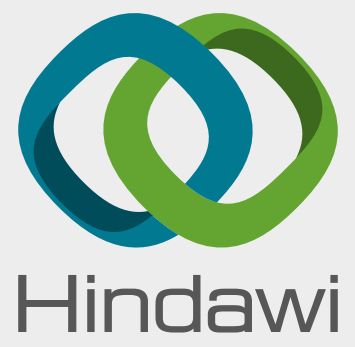

Submit your manuscripts at

www.hindawi.com
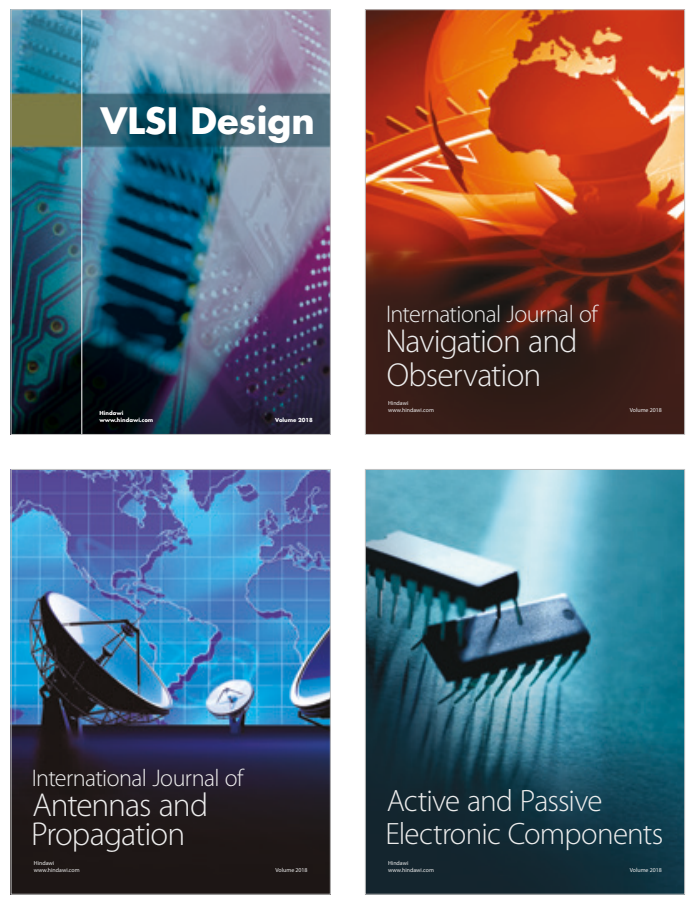
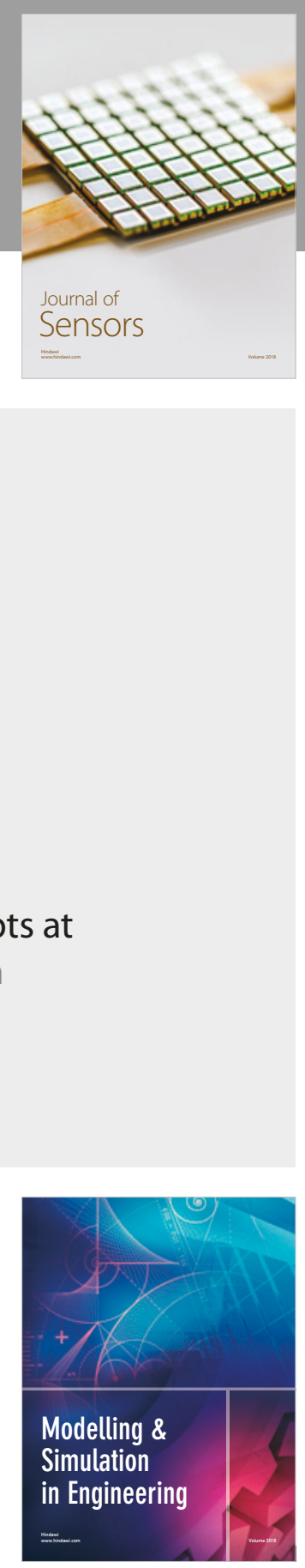

\section{Advances \\ Multimedia}
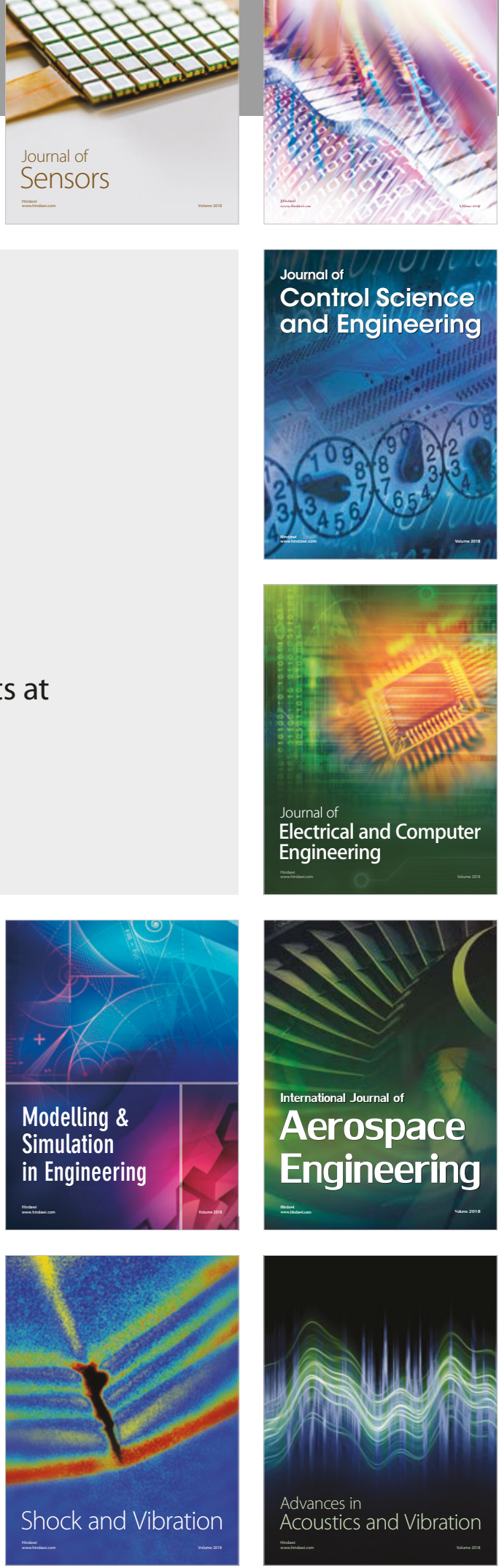